\title{
Sorbitol dehydrogenase inhibitor protects the liver from ischemia/reperfusion-induced injury via elevated glycolytic flux and enhanced sirtuin 1 activity
}

\author{
CHANGHE ZHANG ${ }^{1,2}$, XIANGCHENG $\mathrm{LI}^{2}$ and QINHONG LIU ${ }^{1}$ \\ ${ }^{1}$ Department of General Surgery, Taizhou People's Hospital, Taizhou, Jiangsu 225300; ${ }^{2}$ Department of Liver Transplantation, \\ The First Affiliated Hospital of Nanjing Medical University, Nanjing, Jiangsu 210029, P.R. China
}

Received January 12, 2014; Accepted June 26, 2014

DOI: $10.3892 / \mathrm{mmr} .2014 .2715$

\begin{abstract}
Sorbitol dehydrogenase (SDH), a key enzyme of the polyol pathway, has recently been demonstrated to have an important role in mediating tissue ischemia/reperfusion (I/R) injury. The present study investigated how this enzyme may affect the ischemic liver and the mechanism underlying its effect. Firstly, C57BL/6 mice were subjected to oral administration of CP-470,711 (5 mg/kg body weight/day for five days) and $70 \%$ hepatic I/R. Next the present study further investigated the changes in liver function, histology, inflammation, apoptosis and necrosis; the cytosolic adenosine triphosphate (ATP) and nictotinamide adenine dinucleotide [NAD $(\mathrm{H})]$ contents and the protein level of caspase 3 and sirtuin 1 (SIRT1). The data demonstrated that sorbitol dehydrogenase inhibitor (SDI)-administration significantly alleviated I/R-induced liver injury, palliated histological changes and lowered the level of hepatocyte apoptosis and necrosis. In addition, SDI-pretreatment in ischemic liver markedly maintained the cytosolic ATP and NAD $(\mathrm{H})$ proportion, enhanced SIRT1 and suppressed the activation of caspase 3 at the protein level. The findings in the present study revealed that the flux through $\mathrm{SDH}$ may render the liver more vulnerable to I/R-induced injury and interventions targeting this enzyme may provide a novel adjunctive approach to protect from severe tissue injury following liver ischemia.
\end{abstract}

Correspondence to: Dr Qinghong Liu, Department of General Surgery, Taizhou People's Hospital, 210 Yingchun Road, Taizhou, Jiangsu 225300, P.R. China

E-mail: docliuqinghong@sohu.com

Professor Xiangcheng Li, Department of Liver Transplantation, The First Affiliated Hospital of Nanjing Medical University, 140 Guangzhou Road, Nanjing, Jiangsu 210029, P.R. China

E-mail: doclixiangcheng@sohu.com

Key words: ischemia/reperfusion, sorbitol dehydrogenase, nicotinamide adenine dinucleotide, liver, sirtuin 1, adenosine triphosphate

\section{Introduction}

Ischemia-reperfusion (I/R) injury is a common occurrence in liver surgery. Patients undergoing liver transplantation may encounter severe postoperative complications, namely primary liver dysfunction or non-function despite the long waiting lists and high rate of mortality in those awaiting a compatible donor (1). In addition, patients subjected to major hepatectomy also have a high risk of developing liver dysfunction, in which portal triad occlusion is routinely utilized to reduce blood loss (2). Among the multiple pathogeneses accounting for these complications, I/R induced injury is widely accepted as an important contributor $(1,2)$.

Sorbitol dehydrogenase (SDH), a key enzyme in the polyol pathway, has traditionally been implicated in the development of vascular and neurological complications in diabetes. In this pathway, SDH converts sorbitol to fructose with the concomitant reduction of coenzyme nictotinamide adenine dinucleotide (NAD) to NADH (3). Previously, accumulating evidence has demonstrated the beneficial impact of a sorbitol dehydrogenase inhibitor (SDI) on ischemic tissue (3-5). The potential mechanisms underlying this effect have not been fully elucidated. In addition the role of SDH in the ischemic liver remains unclear. Therefore, the present study aimed to investigate how SDH may affect livers subjected to I/R insult and the mechanism underling its effect.

\section{Materials and methods}

Ethical approval. The present study was approved by the Nanjing Medical University Experimental Animal Department (no. NJMU-AEARIA-4001-20120401; Nanjing, Jiangsu, China) and closely followed the Guide for the Care and Use of Laboratory Animals published by the US National Institutes of Health (NIH publication no. 85-23, revised 1996).

Reagents. SDI (CP-470, 711) was obtained from Pfizer Global Research and Development (Groton, CT, USA). The $\mathrm{NAD}(\mathrm{H})$ and adenosine triphosphate (ATP) Quantification kits were purchased from BioVision (Milpitas, CA, USA). The Annexin V-fluorescein isothiocyanate (FITC)/propidium iodide (PI) apoptosis detection kit was 
purchased from BD Biosciences (Bedford, MA, USA). Interleukin (IL)-1 $\beta$ (catalogue no. MLB00C) and tumor necrosis factor (TNF)- $\alpha$ ELISA assay kits (catalogue no. DY410-05) were obtained from R\&D Systems (Minneapolis, MN, USA). Primary monoclonal rabbit anti-caspase 3 and secondary monoclonal mouse anti-rabbit antibodies were purchased from Cell Signaling Technology, Inc. (Danvers, MA, USA). Primary monoclonal rabbit antibodies against SDH, SIRT1 and $\beta$-actin were purchased from Abcam (Cambridge, MA, USA). The bicinchoninic acid (BCA) protein assay kit were purchased from the Beyotime Institute of Biotechnology (Nanjing, China).

Animals and I/R protocol. Wild-type C57BL/6 male mice were obtained from the Chinese Academy of Sciences (Shanghai, China), maintained in $25 \pm 2^{\circ} \mathrm{C}$ under a $12 \mathrm{~h}$ dark/light cycle. The mice were randomized into three groups containing ten animals each: (i) The SDI group, administered CP-470,711 ( $5 \mathrm{mg} / \mathrm{kg}$ body weight/day) by gavage for five days and subjected to $70 \%$ liver I/R; (ii) the control group, treated with isoquant saline control and subjected to $70 \%$ liver I/R; (iii) the sham group received isoquant CP-470,711 by gavage and sham operation. The usage of CP-470,711 was determined with reference to previous studies $(6,7)$. A total of $24 \mathrm{~h}$ following the last drug treatment, $50 \%$ of the animals from each group underwent $1 \mathrm{~h}$ of $70 \%$ hepatic ischemia as previously described (8) and ischemic liver lobes were harvested to examine the real-time intrahepatic energy status. The other five mice from each group were subjected to an additional $24 \mathrm{~h}$ of reperfusion to detect hepatic tissue impairment and changes in protein levels. The blood samples from mice that had undergone I/R were collected to investigate the levels of inflammatory markers. The animals were eventually sacrificed under deep anesthesia induced by peritoneal chlorali hydras ( $0.6 \mathrm{~g} / \mathrm{kg}$ body weight).

Liver function test. The blood samples were immediately centrifuged at 2,000 $\mathrm{x}$ g to obtain serum following collection. The serum alanine aminotransferase (ALT) and aspartate aminotransferase (AST) were then automatically measured on the Aeroset autoanalyzer (Abbott Laboratories, Chicago, IL, USA) and reported in units per liter.

Detecting serum inflammatory markers. The serum levels of IL-1 $\beta$ and TNF- $\alpha$ were investigated in a 96 -well microtitre plate using a commercial enzyme-linked immunosorbent assay kit according to the manufacturer's instructions. The lower limits of IL-1 $\beta$ and TNF- $\alpha$ detection were 2.31 and $1.88 \mathrm{pg} / \mathrm{ml}$, respectively.

Morphological observations. The histological sections stained with hematoxylin and eosin (H\&E) were processed routinely. The degree of hepatic necrosis and inflammation was evaluated by an experienced pathologist using the Suzuki score, which evaluated the extent of congestion, vacuolization and necrosis on a four-point scale for a total score of $0-12$ as previously described (9).

Flow cytometric analysis of the proportion of hepatocytes undergoing necrosis and apoptosis. Single-hepatocyte suspensions were prepared from the fresh liver using an enzymatic technique as previously described (10). Cells were exposed to $0.04 \%$ Trypan blue for 3 minutes and then observed under a light microscope. Trypan Blue exclusion demonstrated that the cell viability in all of the samples exceeded $89 \%$. The degree of apoptosis and necrosis in the hepatocytes was quantified by flow cytometry using an Annexin V-FITC kit and FlowJo software (Tree Star Inc., Ashland, OR, USA) as previously described (11). The cell percentage in the upper right, upper left and lower right quadrants represented the proportions of late apoptotic, necrotic and early apoptotic cells, respectively. The total apoptosis ratio was then calculated by addingthe proportions of late and early apoptotic cells.

Measuring cytosolic ATP and $N A D(H)$ contents. The hepatic contents of $\mathrm{NAD}(\mathrm{H})$ and the NAD/NADH ratio were determined using $\mathrm{NAD}^{+} / \mathrm{NADH}$ Quantification Kit (BioVision). Briefly, the liver samples were homogenized in extraction buffer and centrifuged at $12,000 \mathrm{xg}$ for $5 \mathrm{~min}$. The supernatant was filtered through $10-\mathrm{kDa}$ Spin Columns (Catalogue no. 1997-25; BioVision) to remove the NADH-consuming enzyme NADase and was then measured according to the manufacturer's instructions.

The ATP content in the hepatocytes was detected by a colorimetric method using a ATP Colorimetric/Fluorometric Assay kit (BioVision), which consumes ATP to generate glycerol 3-phosphate, according to the manufacturer's instructions. To remove the innate ATP-consuming enzymes, the homogenized samples $(10 \mathrm{mg})$ were filtered through the aforementioned spin column prior to being inoculated into 96-well plates. Measurements were performed according to the manufacturer's instructions.

Immunoblotting assay. Total protein from the liver tissues following $\mathrm{I} / \mathrm{R}$ insult were analyzed by immunoblotting as previously described (12). Prior to sample-loading, the BCA Protein Assay kit was conventionally used to determine the homogeneity of protein concentrations in different columns. The primary antibodies were used at the following concentrations: Anti-SDH (1:1,500), -caspase-3 (1:2,000), -SIRT1 $(1: 2,000)$ and $-\beta$-actin $(1: 2,500)$. The relative protein levels were determined using Image J software (NIH, Bethesda, MD, USA). $\beta$-actin served as a reference protein

Statistical analysis. Data are expressed as the mean \pm standard deviation. Statistical analysis of the data was performed using the Duncan's test with SPSS 16.0 software (SPSS, Inc., Chicago, IL, USA). P $<0.05$ was considered to indicate a statistically significant difference. All of the results were obtained from at least five independent experiments.

\section{Results}

SDI protects mouse liver against I/R-induced impairment. The transaminases ALT and AST are generated innately within hepatocytes and marked elevations in serum levels denote hepatocellular membrane leakage caused by inflammation and/or necrosis. In the present study, I/R markedly increased the serum transaminase levels, whereas SDI-pretreatment markedly ameliorated these changes (Fig. 1A). 

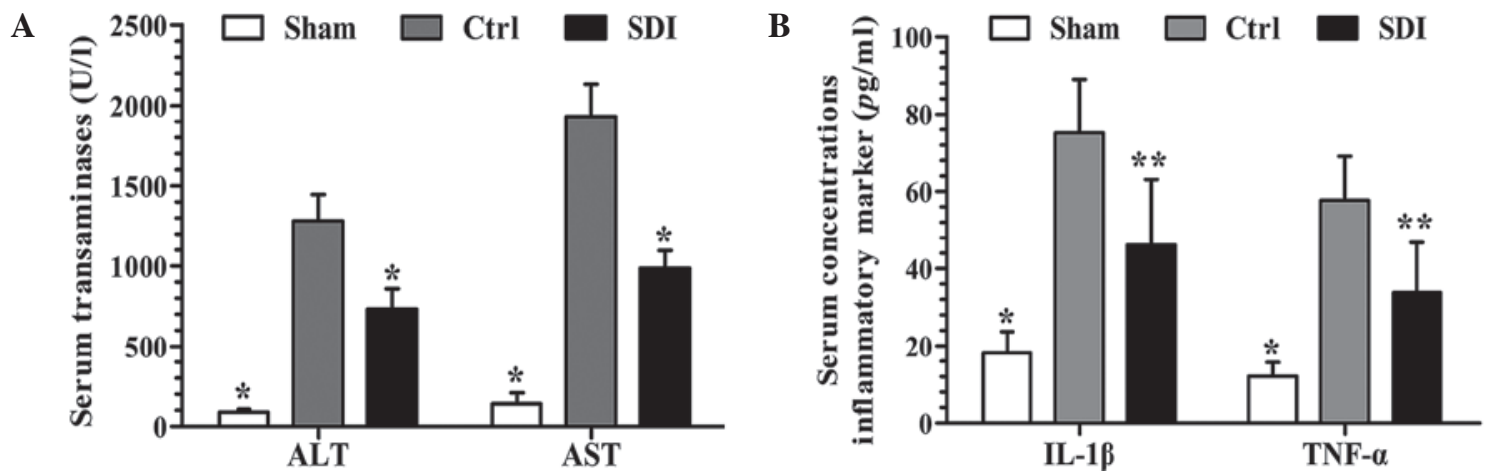

Figure 1. SDI protected mice liver against I/R-induced injury. (A) I/R markedly increased the serum transaminase levels, whereas SDI-pretreatment notably palliated these changes. (B) ARI markedly alleviated the I/R-mediated serum IL- $1 \beta$ and TNF- $\alpha$ elevation as compared with the $\mathrm{Ctrl}$ group. $\mathrm{n}=5$; ${ }^{*} \mathrm{P}<0.01$ and ${ }^{* *} \mathrm{P}<0.05$ as compared with the Ctrl group. SDI, sorbitol dehydrogenase; I/R, ischemia/reperfusion; ARI, ; IL-1 $\beta$, interleukin-1 $\beta$; TNF- $\alpha$, tumor necrosis factor- $\alpha$; Ctrl, control.

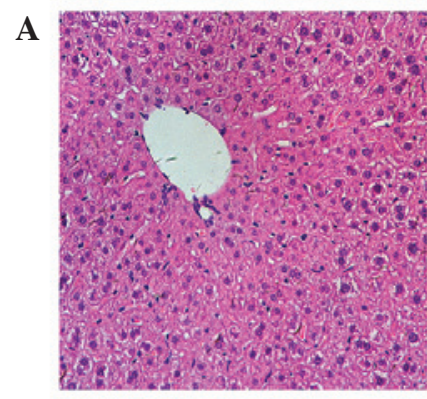

Sham

C

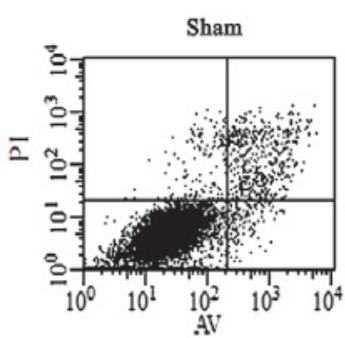

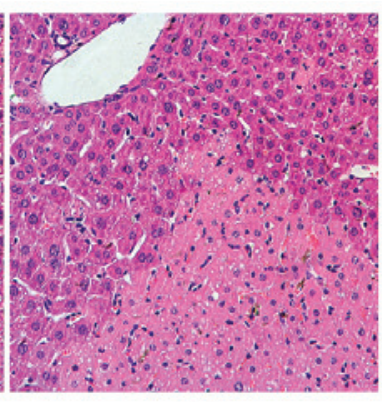

Ctrl

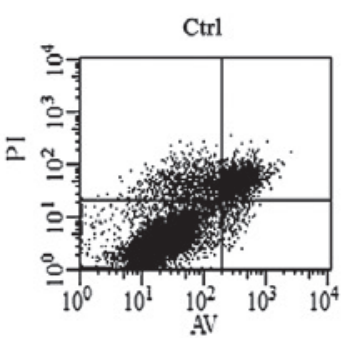

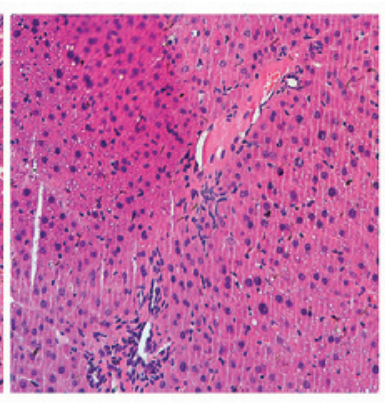

SDI

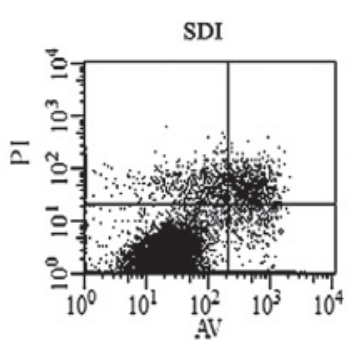

B

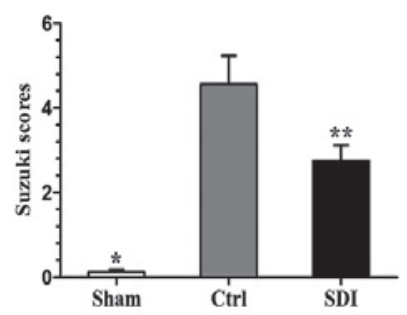

D

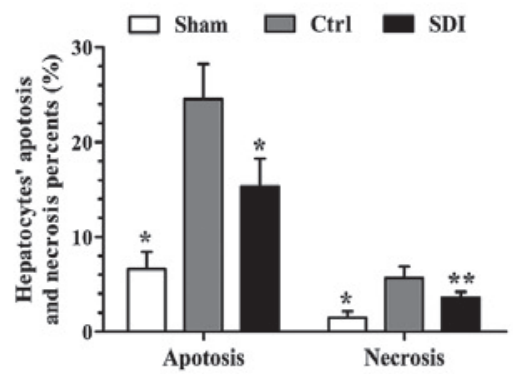

Figure 2. SDI-pretreatment normalized liver histology and improved hepatocyte fate. (A and B) In hematoxylin and eosin staining sections (magnification, $\mathrm{x} 400$ ), the I/R insult significantly disrupted liver histology and contributed to evident necrosis, while SDI markedly prevented these changes. (B) The Ctrl group demonstrated a higher Suzuki score than the sham group and this trend was mitigated by SDI. (C and D) In flow cytometry, the proportions of apoptotic and necrotic hepatocytes in the Ctrl group were significantly higher than in the sham group, whereas SDI inhibited these changes. $\mathrm{n}=5$; ${ }^{*} \mathrm{P}<0.01$ and ${ }^{* *} \mathrm{P}<0.05$ as compared with the Ctrl group. SDI, sorbitol dehydrogenase; I/R, ischemia/reperfusion; Ctrl ,control; AV, Annexin V; PI, propidium iodide.

IL-1 $\beta$ and TNF- $\alpha$ are widely accepted to be inflammatory markers and an increase in the serum level indicates a severe inflammatory reaction and tissue injury (13). As demonstrated in Fig. 1B, SDI administration markedly alleviated the I/R-mediated elevation in the serum level of IL- $1 \beta$ and TNF- $\alpha$ as compared with the control group.

\section{SDI-pretreatment prevents the I/R-induced histological} changes and improves hepatocyte fate. As direct causes of liver dysfunction following I/R, hepatic inflammation, apoptosis and necrosis were analyzed in the present study. As demonstrated in Fig. 2A, I/R insult significantly disrupted liver histology and contributed to significant necrosis in contrast to the sham group, where SDI markedly prevented the changes in hepatic histology. Consistently, the quantitative Suzuki score in the control group was also evidently higher than that in sham group and this trend was significantly mitigated by SDI treatment (Fig. 2B). In the flow cytometric analysis, the proportions of apoptotic and necrotic hepatocytes in the control group were higher than the sham group, while SDI significantly reverted these changes (Fig. 2C and D).

SDI reverses the I/R-mediated NAD/NADH imbalance and energy deficit. The coenzyme $\mathrm{NAD}(\mathrm{H})$ participates in the polyol and glycolysis pathways, the latter of which acts as a major ATP source in the ischemic liver $(3,14,15)$. Therefore, the present study analyzed the $\mathrm{NAD}(\mathrm{H})$ and ATP content in 

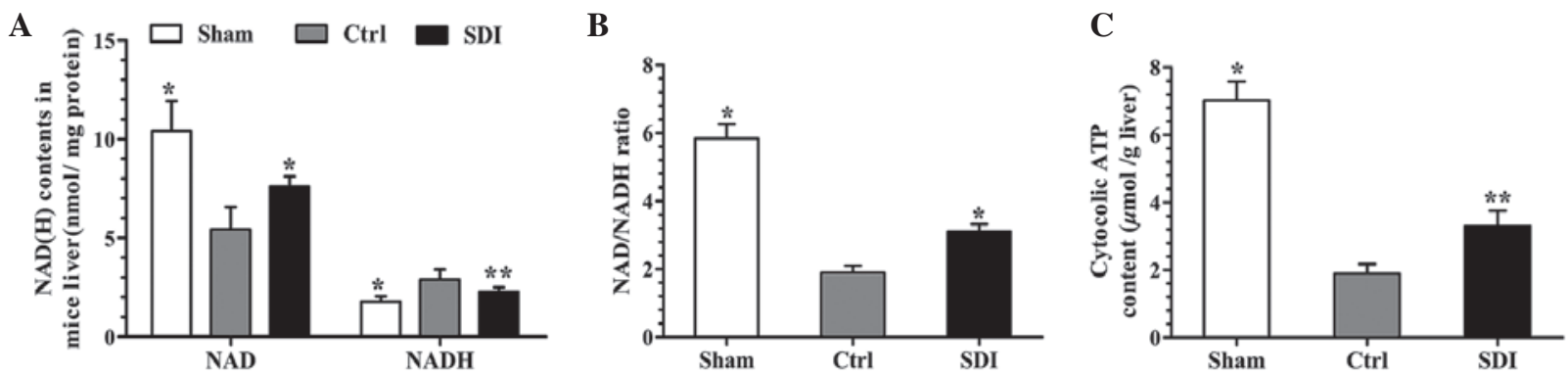

Figure 3. SDI reversed the I/R-mediated NAD/NADH imbalances and ameliorated hepatic energy status. (A-C) SDI significantly alleviated the I/R-induced decrease in the NAD and ATP content meanwhile reversed NAD/NADH imbalance. $\mathrm{n}=5$; ${ }^{*} \mathrm{P}<0.01$ and ${ }^{* * *} \mathrm{P}<0.05$ as compared with the Ctrl group. SDI, sorbitol dehydrogenase; I/R, ischemia/reperfusion; Ctrl, control.

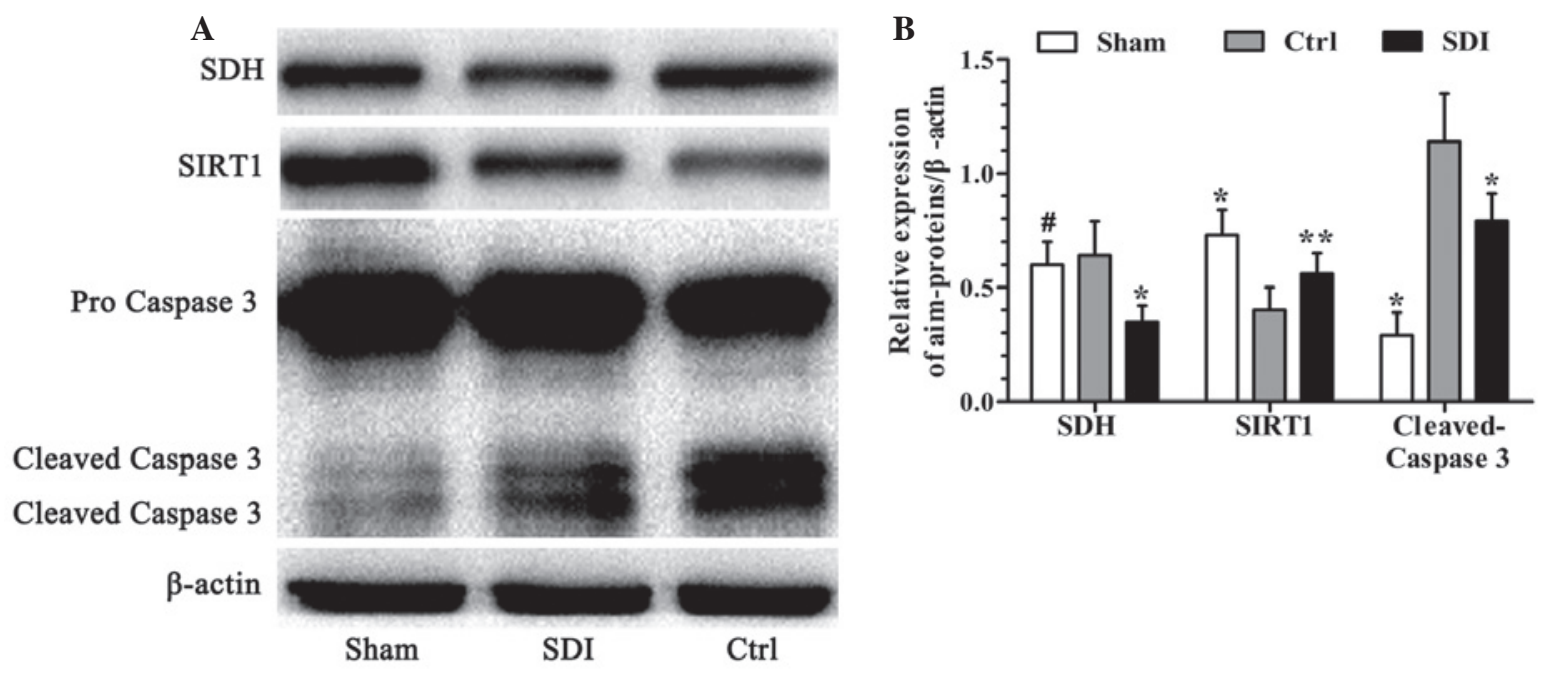

Figure 4. SDI enhanced SIRT1 and inhibited caspase 3 activation in liver following I/R. (A and B) In protein level, I/R suppressed SIRT1 and enhanced caspase 3 whereas SDI palliated the trends. Additionally, the sham and Ctrl groups exhibited a nearly identical level of SDH protein which was markedly higher than the SDI group. $\mathrm{n}=5 ;{ }^{*} \mathrm{P}<0.01,{ }^{* *} \mathrm{P}<0.05$ and ${ }^{\#} \mathrm{P}>0.05$ as compared with the Ctrl group. SDI, sorbitol dehydrogenase; I/R, ischemia/reperfusion; Ctrl, control.

the ischemic liver, intending to examine the possible causal association between SDI-administration, NAD $(\mathrm{H})$ and ATP content. As demonstrated in Fig. 3A-C, SDI significantly alleviated the I/R-induced decrease in NAD and ATP content and reversed the NAD/NADH imbalance compared with the control group.

SDI enhances SIRT1 protein expression and inhibits caspase 3 in the liver following $I / R$. As an important cellular function regulator and an NAD-dependent enzyme, SIRT1 deacetylase was quantified by an immunoblotting assay (16). As demonstrated in Fig. 4A and B, I/R markedly suppressed SIRT1 at the protein level while SDI alleviated this change.

It is well-established that caspase 3 acts as a major and common executor in multiple apoptotic pathways when cells are exposed to cytotoxic stressors, including I/R $(17,18)$. The level of cleaved caspase 3 was then measured using western blotting. As demonstrated in Fig. 4A and B, SDI markedly suppressed the I/R-induced activation of protein caspase 3 as compared with the control group.

Additionally, SDH protein was also quantified to assess the inhibition efficiency of SDI. In the present study, the sham and control groups exhibited a similar level of SDH protein, which was notably higher than that in the SDI group (Fig. 4A and B).

\section{Discussion}

In the present study, it was firstly demonstrated that SDI markedly alleviated liver I/R-induced impairment and improved hepatocyte fate. These findings are consistent with those of previous studies investigating other target organs (3-5). The changes in $\mathrm{NAD}(\mathrm{H})$ content and proportion were markedly ameliorated following SDI administration, providing evidence that these beneficial effects may be associated with the improved co-enzyme balance and resultant cellular metabolic and signaling adaptations.

Under hypoxic conditions, low levels of ATP are generated within the liver by glycolysis to sustain basic cellular function $(14,15,19)$. In the glycolysis pathway, the key enzyme GAPDH exerts a catalytic function dependent upon the synchronous reduction of NAD to NADH, which denotes a competitive antagonistic correlation between SDH and GAPDH due to the limited quantity of NAD $(20,21)$. Therefore, it is reasonable to hypothesize that inhibiting SDH may prevent this competition for substrate and enhance the glycolysis flux. This hypothesis has been widely validated by previous studies based on models of ischemic heart, brain and kidney injury. In these studies, the reduction in the levels of NAD and/or the NAD/NADH ratio was caused 
by niacin deficiency or poly (ADP-ribose) polymerase overactivation, which clearly aggravated tissue I/R injury. The ARI/SDI pretreatment or NAD/niacin repletion markedly reversed this phenomenon (3-5,22-25). In the present study, pretreating ischemic mice with SDI also contributed to an evident elevation in the NAD/NADH ratio and ATP content. These results were consistent with the findings of previous studies $(3,4,22-24)$.

Hepatocyte inflammation, apoptosis and necrosis represent different extremes in a continuum of cellular responses to severe stressors $(17,18)$. The major difference between them is that inflammation refers mainly to the reaction of cells to stimuli, while apoptosis and necrosis are cell death processes. However, apoptosis is an active, ATP-consuming event, while necrosis is passive, without ATP participation $(13,18)$. In the present study, intracellular ATP content was significantly increased following SDI administration. This may explain why histology and flow cytometry in the SDI group primarily demonstrated inflammation and apoptosis, whereas the control group demonstrated evidence of apoptosis and necrosis.

Aside from GAPDH, SIRT1 deacetylase represents another NAD-dependent enzyme. SIRT1 is a member of the mammalian sirtuin family and widely participates in cellular death modulating activities, including mitochondrial function, poly (ADP-ribose) polymerase-1 (PARP-1) cytotoxicity and apoptotic effects $(20,22,26-29)$. Therefore, due to its roles in delaying aging and anti-apoptosis, SIRT1 is generally acknowledged to be a lifespan promoter, preventing cell death. In the present study, SIRT1 protein expression was suppressed by I/R injury, while SDI notably inhibited this trend. Limited by the scale of the study, the specific role of SIRT1 in the ischemic liver was not investigated. However, it is hypothesized that the elevated SIRT1 flux may contribute to reduced I/R-induced liver injury.

Accumulating evidence has suggested that NAD is a fundamental mediator of various biological processes (20). Apart from the aforementioned regulative activities, an appropriate proportion of $\mathrm{NAD}(\mathrm{H})$ is required to maintain mitochondrial function and cellular calcium homeostasis (3,20,30-32). Furthermore, NAD also has a pivotal role in activating the PARP-1-dependent death pathway $(22,25,33)$. Therefore, a paradoxical theme emerges: The SDI-induced NAD elevation may protect the ischemic liver through improved glycolysis and/or SIRT1 activities; conversely, increased cytosolic NAD content may contribute to elevated PARP-1-mediated hepatocyte apoptosis. Considering the variance in liver function, histology and hepatocyte fate, it was hypothesized that SDI predominantly exhibits a beneficial role in hepatic I/R injury. However, for potential clinical consideration, further, more comprehensive studies are required.

In conclusion, the present study demonstrated that SDI protects the liver against I/R-induced injury in terms of improved liver function, ameliorated histopathological changes and reduced levels of hepatic necrosis and apoptosis. This effect may be further associated with an elevated NAD/NADH ratio and resultant elevation in glycolysis flux and SIRT1 expression. The present study suggests that SDI may be used clinically to protect the liver from severe tissue impairment following I/R injury.

\section{Acknowledgements}

The present was fully financed by the National Natural Science Foundation of China (grant no. 81170415).

\section{References}

1. Elias-Miró M, Massip-Salcedo M, Raila J, et al: Retinol binding protein 4 and retinol in steatotic and nonsteatotic rat livers in the setting of partial hepatectomy under ischemia/reperfusion. Liver Transpl 18: 1198-1208, 2012.

2. Varona MA, Soriano A, Aguirre-Jaime A, et al: Statistical quality control charts for liver transplant process indicators: evaluation of a single-center experience. Transplant Proc 44: 1517-1522, 2012.

3. Tang WH, Wu S, Wong TM, Chung SK and Chung SS: Polyol pathway mediates iron-induced oxidative injury in ischemic-reperfused rat heart. Free Radic Biol Med 45: 602-610, 2008.

4. Li Q, Hwang YC, Ananthakrishnan R, et al: Polyol pathway and modulation of ischemia-reperfusion injury in Type 2 diabetic BBZ rat hearts. Cardiovasc Diabetol 7: 33, 2008.

5. Tang WH, Kravtsov GM, Sauert M, et al: Polyol pathway impairs the function of SERCA and RyR in ischemic-reperfused rat hearts by increasing oxidative modifications of these proteins. J Mol Cell Cardiol 49: 58-69, 2010.

6. Chu-Moyer MY, Ballinger WE, Beebe DA, et al: SAR and species/stereo-selective metabolism of the sorbitol dehydrogenase inhibitor, CP-470,711. Bioorg Med Chem Lett 12: 1477-1480, 2002.

7. Schmidt RE, Dorsey DA, Beaudet LN, et al: A potent sorbitol dehydrogenase inhibitor exacerbates sympathetic autonomic neuropathy in rats with streptozotocin-induced diabetes. Exp Neurol 192: 407-419, 2005.

8. Ramalho FS, Alfany-Fernandez I, Casillas-Ramirez A, et al: Are angiotensin II receptor antagonists useful strategies in steatotic and nonsteatotic livers in conditions of partial hepatectomy under ischemia-reperfusion? J Pharmacol Exp Ther 329: 130-140, 2009.

9. Suzuki S, Toledo-Pereyra LH, Rodriguez FJ and Cejalvo D: Neutrophil infiltration as an important factor in liver ischemia and reperfusion injury. Modulating effects of FK506 and cyclosporine. Transplantation 55: 1265-1272, 1993.

10. Berry MN and Friend DS: High-yield preparation of isolated rat liver parenchymal cells: a biochemical and fine structural study. J Cell Biol 43: 506-520, 1969.

11. Wang S, Chen T, Chen R, et al: Emodin loaded solid lipid nanoparticles: preparation, characterization and antitumor activity studies. Int J Pharm 430: 238-246, 2012.

12. Lee HR, Kim HJ, Ko JS, et al: Comparative characteristics of porous bioceramics for an osteogenic response in vitro and in vivo. PLoS One 8: e84272, 2013.

13. Nikoletopoulou V, Markaki M, Palikaras K and Tavernarakis N: Crosstalk between apoptosis, necrosis and autophagy. Biochim Biophys Acta 1883: 3448-3459, 2013.

14. So PW and Fuller BJ: Enhanced energy metabolism during cold hypoxic organ preservation: studies on rat liver after pyruvate supplementation. Cryobiology 46: 295-300, 2003.

15. Katz NR: Metabolic heterogeneity of hepatocytes across the liver acinus. J Nutr 122 (Suppl): 843-849, 1992.

16. Yu Q, Wang T, Zhou X, et al: Wld(S) reduces paraquat-induced cytotoxicity via SIRT1 in non-neuronal cells by attenuating the depletion of NAD. PLoS One 6: e21770, 2011.

17. Bantel $\mathrm{H}$ and Schulze-Osthoff $\mathrm{K}$ : Mechanisms of cell death in acute liver failure. Front Physiol 3: 79, 2012.

18. Malhi H, Gores GJ and Lemasters JJ: Apoptosis and necrosis in the liver: a tale of two deaths? Hepatology 43 (Suppl 1): 31-44, 2006.

19. Brosnan JT, Krebs HA and Williamson DH: Effects of ischaemia on metabolite concentrations in rat liver. Biochem J 117: 91-96, 1970.

20. Ying $\mathrm{W}: \mathrm{NAD}+\mathrm{NADH}$ and $\mathrm{NADP}+\mathrm{NADPH}$ in cellular function and cell death: regulation and biological consequences. Antioxid Redox Signal 10: 179-206, 2008.

21. Dodson M, Darley-Usmar V and Zhang J: Cellular metabolic and autophagic pathways: traffic control by redox signaling. Free Radic Biol Med 63: 207-221, 2013.

22. Ying W, Garnier P and Swanson RA: NAD+ repletion prevents PARP-1-induced glycolytic blockade and cell death in cultured mouse astrocytes. Biochem Biophys Res Commun 308: 809-813, 2003. 
23. Hwang YC, Kaneko M, Bakr S, et al: Central role for aldose reductase pathway in myocardial ischemic injury. FASEB J 18: 1192-1199, 2004.

24. Ramasamy R, Trueblood N and Schaefer S: Metabolic effects of aldose reductase inhibition during low-flow ischemia and reperfusion. Am J Physiol 275: H195-H203, 1998.

25. Benavente CA and Jacobson EL: Niacin restriction upregulates NADPH oxidase and reactive oxygen species (ROS) in human keratinocytes. Free Radic Biol Med 44: 527-537, 2008.

26. Dong W, Li F, Pan Z, et al: Resveratrol ameliorates subacute intestinal ischemia-reperfusion injury. J Surg Res 185: 182-189, 2013.

27. Yan W, Fang Z, Yang Q, et al: SirT1 mediates hyperbaric oxygen preconditioning-induced ischemic tolerance in rat brain. J Cereb Blood Flow Metab 33: 396-406, 2013.

28. Lempiäinen J, Finckenberg P, Mervaala EE, et al: Caloric restriction ameliorates kidney ischaemia/reperfusion injury through PGC-1 $\alpha$-eNOS pathway and enhanced autophagy. Acta Physiol (Oxf) 208: 410-421, 2013.
29. Pillai JB, Isbatan A, Imai S and Gupta MP: Poly (ADP-ribose) polymerase-1-dependent cardiac myocyte cell death during heart failure is mediated by NAD+ depletion and reduced Sir2alpha deacetylase activity. J Biol Chem 280: 43121-43120, 2005.

30. Alano CC, Ying W and Swanson RA: Poly (ADP-ribose) polymerase-1-mediated cell death in astrocytes requires $\mathrm{NAD}^{+}$ depletion and mitochondrial permeability transition. J Biol Chem 279: 18895-18902, 2004.

31. Jang SY, Kang HT and Hwang ES: Nicotinamide-induced mitophagy: event mediated by high $\mathrm{NAD}^{+} / \mathrm{NADH}$ ratio and SIRT1 protein activation. J Biol Chem 287: 19304-19314, 2012.

32. Mouchiroud L, Houtkooper RH, Moullan N, et al: The $\mathrm{NAD}^{+} /$sirtuin pathway modulates longevity through activation of mitochondrial UPR and FOXO signaling. Cell 154: 430-441, 2013.

33. Siegel $\mathrm{C}$ and McCullough LD: $\mathrm{NAD}^{+}$depletion or PAR polymer formation: which plays the role of executioner in ischaemic cell death? Acta Physiol (Oxf) 203: 225-234, 2011 